\section{3 '2' 1: Orthodontic re-positioning of canines into central incisors}

\author{
A. Mahdmina, ${ }^{1}$ 0. Malik, ${ }^{2}$ M. Ashley ${ }^{3}$ and D. Waring ${ }^{4}$ \\ VERIFIABLE CPD PAPER
}
IN BRIEF
- Outlines aetiology, special investigations, risk factors and treatment options for impacted maxillary canines.
- Considers risk associated with impacted maxillary canines.
- Outlines importance of early detection and early interceptions.
- Demonstrates how the impacted canine can be successfully camouflaged as the resorbed central incisor giving excellent functional and aesthetic results.

Resorption of lateral incisors caused by impacted maxillary canines is frequently reported. However, resorption of the central incisor is less common and management of such a finding can prove to be a challenge for the clinician. This article reviews the literature of impacted canines and incisor resorption. The management of two cases of severe central incisor resorption caused by an impacted maxillary canine is also described.

\section{INTRODUCTION}

The maxillary canine is the second most commonly impacted tooth after the mandibular third molar. With the highest incidence reported at 3\%, ${ }^{1}$ the impacted maxillary canine is a relatively common finding and can present many challenging complications for the clinician such as root resorption in the maxillary incisors. Impacted canines are more prevalent in female patients with a ratio of $2: 1$ and most impactions (80\%-92.6\%) are palatal as opposed to buccal., ${ }^{2,3}$ No difference has been found in the frequency of occurrence of the right and left maxillary canines. ${ }^{4}$

\section{AETIOLOGY}

The aetiology is believed to be multifactorial with a combination of local and general factors but most likely due to the long and tortuous path of eruption of the maxillary canine. Local complicating factors include a spaced arch, ${ }^{5}$ crowding and a lack of space, ${ }^{5,6}$ trauma, ${ }^{7}$ and diminutive, absent or unusually placed lateral incisors as with Class II division $2 .^{8,9}$ Spacing in an uncrowded arch or in the presence of hypodontia, along with diminutive or absent lateral incisors, are

'Dental Foundation 2, ${ }^{2}$ Consultant in Orthodontics, ${ }^{3}$ Consultant in Restorative Dentistry, ${ }^{4 *}$ Consultant in Orthodontics, University Dental Hospital of Manchester,

Higher Cambridge Street, Manchester, M15 6FH

*Correspondence to: David Waring

Email: david.waring@manchester.ac.uk

\section{Refereed Paper}

Accepted 11 January 2012

DOI: 10.1038/sj.bdj.2012.318

${ }^{\oplus}$ British Dental Journal 2012; 212: 369-375 explained as possible aetiological factors by the 'guidance theory', where the distal aspect of the root of the maxillary lateral incisor is believed to act as an eruptive guidance path for the maxillary canine. Therefore spacing or abnormally shaped roots may deprive the maxillary canine of this guidance. ${ }^{8,10}$

\section{DETECTION AND PREVENTION}

Recognising an impacted canine early is of paramount importance and all clinicians are encouraged to palpate for the canine in the buccal sulcus at age 8-10, where the canine is expected to be located at the distal aspect of the maxillary lateral incisor root, and also monitor the deciduous canine for mobility. The usual age for exfoliation of the deciduous canine is 11 years \pm 9 months. ${ }^{11}$ Eruption of the maxillary canine should be considered late at age 12.3 years in girls and 13.1 years in boys. ${ }^{12}$ Where the diagnosis is made later than 12-13 years treatment plans are considerably longer and more complex. ${ }^{13}$

Early detection can prevent lengthy treatment plans as preventive measures such as extraction of the deciduous canine have been shown, in one study, to result in $78 \%$ of palatally placed canines to assume a normal position in 10-13-yearolds. Furthermore this study demonstrated that when the canine crown is distal to the midline of lateral incisors at the time of extraction this rate can increase to as much as 91\%. ${ }^{14}$ However, Power and Short demonstrated that this success rate is significantly lowered where there is crowding of the arch; therefore all aspects of the malocclusion must be considered. ${ }^{15}$

Another preventive measure suggested is the surgical exposure of the superficial and buccally impacted canine which may be enough to induce eruption. ${ }^{16,17}$ Clinicians must be vigilant in detection and alerting dental factors include agenesis, dental malformation, short roots, taurodontism, invaginations and abnormal eruption. These factors are associated with an increased risk of root resorption during orthodontic treatment and may also play a role in resorption associated with impacted canines. ${ }^{18}$

Interestingly one study also found that $23.3 \%$ of patients with ectopic eruption of the first maxillary molar causing root resorption of the maxillary second molar had later developed resorption of the maxillary incisors secondary to maxillary canine impaction. This indicates that there may be a genetic factor predisposing certain individuals to ectopic eruption and root resorption. An ectopic first molar eruption could be another early warning that ectopic canine eruption could follow causing root resorption in the maxillary incisors. ${ }^{19}$

\section{RADIOGRAPHIC INVESTIGATIONS}

The optimal age for radiographic investigation is at age 10-13 depending on somatic development. Radiographic investigation is indicated where:

1. There is asymmetry between the right and left side on palpation of the buccal sulcus 


\section{PRACTICE}

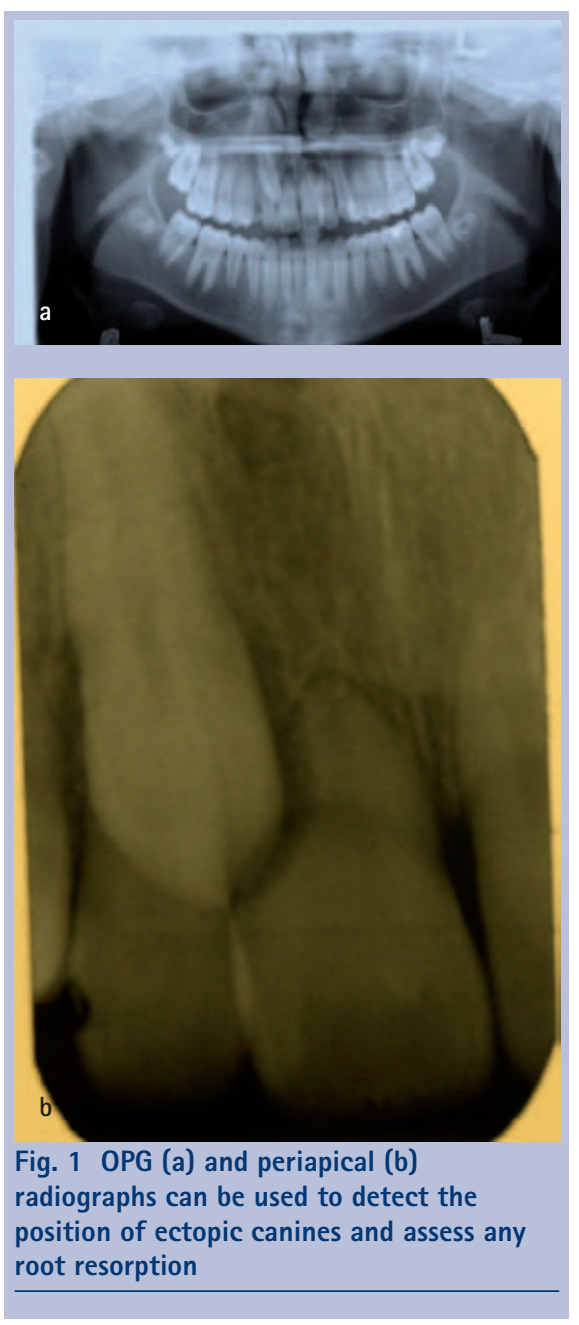

2. Canines are not palpable in the normal site despite normal occlusal development otherwise

3. Maxillary lateral incisors are late in eruption or are proclined.

The selection of radiographs is a significant factor in the diagnosis of impaction and resorption and the stepwise radiographic procedure described by Ericson and $\mathrm{Kurol}^{2}$ has been shown to double the detection rate of impacted maxillary canines. ${ }^{20}$ In the stepwise assessment step one includes 2-3 periapicals, vertex axial, an orthopantograph and lateral cephalogram where extensive orthodontic treatment will be required (Fig. 1). In 92\% of cases the correct position of the canine can be detected using periapical radiographs alone. Where this is not possible and where there is suspicion of resorption from the baseline radiographs, for example due to overlapping of the canine and the lateral incisor, computed tomography may also be indicated (Figs 2a and b).

Where resorption is suspected the degree of overlapping, interruptions in the lamina
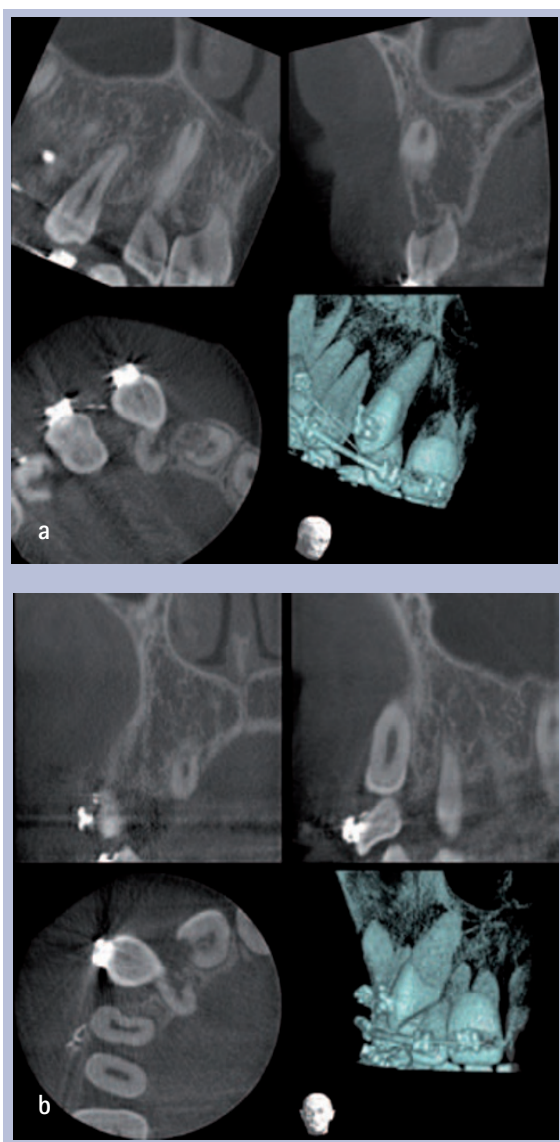

Figs 2a and $b$ A cone beam CT scan (of another patient) provides a detailed position of the impacted radiograph and the relationship to surrounding structures

dura structure and the root contour should also be checked. ${ }^{2}$

\section{Computed tomography}

Computed tomography has been shown to be an important tool in assessing the impacted canines and presence of resorption $^{3,4,13,21}$ (Figs 3a and b). One study showed that $43.7 \%$ of treatment plans for 80 children with retained maxillary deciduous canines were changed after a cone beam computed tomography (CBCT) scan was taken (as opposed to plain radiographs alone). Furthermore, some treatment plans were changed from extraction to nonextraction or where resorption was present, the resorbed tooth was planned to be removed as opposed to the premolar as first planned using plain radiographs alone. ${ }^{13}$

CBCTs accurately show:

1. The presence or absence of the canine

2. Size of the canine follicle

3. Inclination of the long axis of the tooth

4. Relative buccal/palatal position

5. Bone quantity covering the tooth
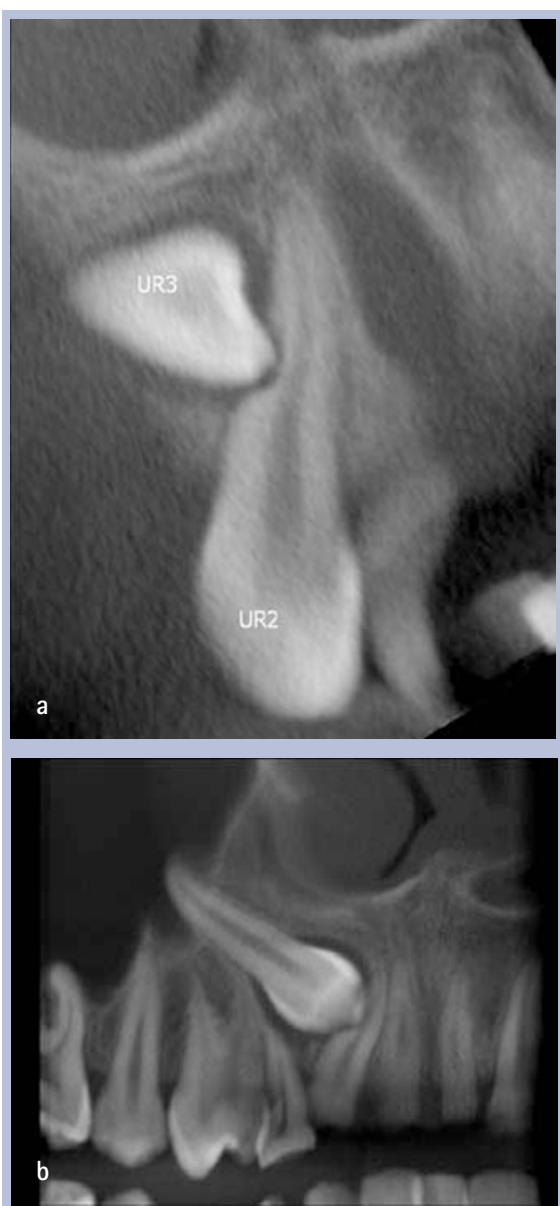

Figs $3 a$ and $b$ The cone beam CT (СBCT) reveals the full extent of resorption with a 3-dimensional view. The CBCT in this case changed the eventual treatment plan as both the lateral and central incisor had a very poor long term prognosis
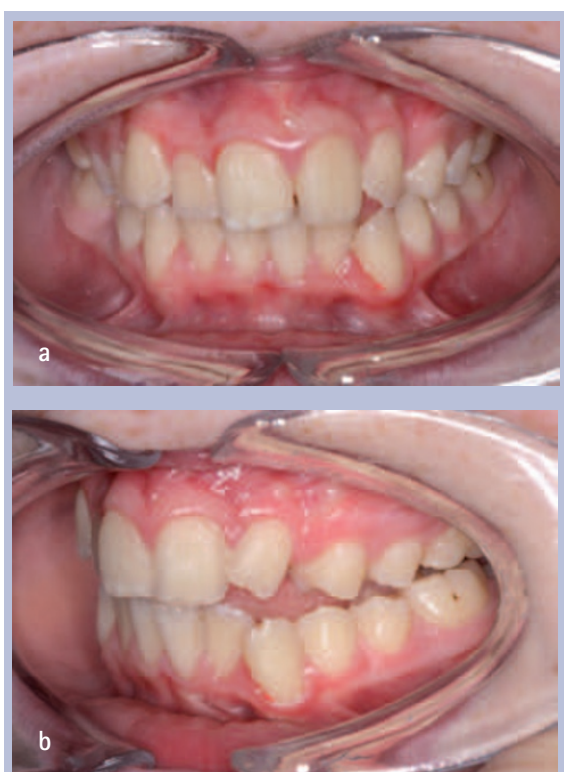

Figs $4 a$ and $b$ Intra-oral pictures demonstrating a Class I incisor relationship with upper and lower crowding. The upper left canine (UL3) is unerupted and in an ectopic position. There is an upper centre line discrepancy 
6. Proximity and resorption of adjacent roots

7. Condition of adjacent teeth

8. Any anatomical considerations

9. Overall dental development stage. ${ }^{3}$

Detection rate of resorption by means of CBCT is $38 \% \%^{4}$ in comparison to radiographs alone where this rate is $12.5 \% .^{20}$ CBCTs therefore detect around 50\% more root resorption on adjacent teeth compared with conventional radiographs where even severe resorption involving the pulp may not be apparent. One study showed ten cases of resorption out of 34 lateral incisors with severe resorption to be completely undetected on intraoral radiographs. ${ }^{4}$ It has also been demonstrated that a close correlation exists between the CBCT image and direct observation of the resorbed tooth following extraction. ${ }^{22}$

Another important advantage of CBCT is the elimination of blurring thereby increasing detection of resoprtion. ${ }^{23}$ It is also important to note that there is a degree of overlapping in $45 \%$ of cases where the canine is assessed by means of intraoral radiographs and a CBCT not only shows the resorption but also other lesions which are not always detected on intraoral radiographs. ${ }^{2}$ Despite the many advantages, however, the large radiation dose of CBCT must always be considered and careful selection criteria are required. A large volume CBCT can have an effective dose of up to $368 \mu \mathrm{Sv}^{24}$ in comparison to intraoral and panoramic radiographs which have effective doses of $<8.3 \mu \mathrm{Sv}^{25}$ and $2.7-23 \mu \mathrm{Sv}^{26}$ respectively.

\section{RESORPTION}

Amongst the many complications of impacted maxillary canines, root resorption of adjacent teeth is not uncommon. External resorption can be described as the loss of dentine or cementum from the outer surface of the root of a tooth. ${ }^{27}$

Resorption is usually asymptomatic ${ }^{20,28}$ and found in $12 \%$ of incisors adjoining ectopic maxillary canines giving an overall prevalence of $0.7 \%$ in 10-13-year-olds. ${ }^{2}$ A correlation has not been found between age, gender and resorption ${ }^{4}$ although cases of central and lateral incisor resorption seem to be more common in boys than girls. ${ }^{19}$ The most common age of resorption is at 11-12 years old. However, severe resorption has been found in patients aged as young as 9 years old. Resorption is more common than previously thought, affecting $48 \%$ of patients with ectopic canines ${ }^{4}$ and is thought to occur more frequently in connection with palatally impacted canines. ${ }^{19}$ However, other studies ${ }^{29,30}$ have also demonstrated that resorption does occur in buccally placed maxillary canines and therefore one must be vigilant and give consideration to resorption in all cases of ectopic canines.

\section{Predisposing factors}

Resorption in permanent teeth is a complex biological process and the exact causative mechanism of root resorption is not yet clear but a combination of local and genetic factors are thought to be responsible. A genetic component ${ }^{28}$ could lead to certain positions predisposing to resorption. However, it is most likely that resorption is caused in more instances by local factors as most cases of resorption occur unilaterally. ${ }^{20}$ It is believed that the continuous physical pressure exerted from the migration of the misplaced, erupting canine ${ }^{21,28,32}$ is responsible for causing root resorption.

Other contributing factors include normal sized incisor roots, ${ }^{20,28,33}$ close proximity of the canine (usually $<0.5 \mathrm{~mm}$ ) to the adjacent tooth, ${ }^{3,4,28}$ a more developed root formation of the canine, ${ }^{28,31}$ a more medial canine position in the arch and mesial inclination of the canine to the midline exceeding $25^{\circ}$. These factors increased the risk of resorption twofold..$^{31}$

Factors thought to be less significant are the width and shape of the canine follicle, ${ }^{3,20,32}$ crowding, ${ }^{20,31}$ condition or location of the deciduous canine, ${ }^{20}$ width of alveolus, ${ }^{3}$ position of the canine or lateral incisors relative to the dental arch, canine position in the sagittal plane, degree of resorption of the deciduous canine, degree of canine vertical eruption and proclination or distal tilting of the lateral incisor. ${ }^{31}$ Interestingly, Sasakura et al. ${ }^{28}$ found dwarfism of incisors to be unrelated to impacted canines.

\section{Site of occurrence}

Resorption is most commonly seen in lateral incisors (38\%) or central incisors (9\%) but resorption in both teeth simultaneously is more rare. ${ }^{4}$ Lateral incisor resorption is seen more bilaterally whereas central incisor resorption occurs more unilaterally. Resorption in the first premolar has also been seen but is thought to be rare. ${ }^{3}$ It is thought that lateral incisors are predisposed to resorption due to their conical root shape, having an apex deep in the palate near the canine crown, and having susceptible roots during development. ${ }^{34}$ Mostly the middle third (82\%) of the incisors were affected followed by apical thirds (13\%) and cervical third (5\%). ${ }^{20}$ This seems to be related to the vertical position of the canine.

Resorption is seen mostly on the distopalatal and distal surfaces of central incisors and palatal and distopalatal surfaces of the root of lateral incisors. The position and inclination of the lateral incisor does not seem to be related to the extent and location of resorption..$^{20}$ It is known that resorption is a rapid process. ${ }^{35}$ Interestingly, cases of moderate resorption are seen less often and, when examined, the incisor roots tend to be either mildly or severely resorbed; ${ }^{4}$ one study shows most central incisor resorption to be advanced and in lateral incisors resorption was either moderate or advanced. ${ }^{20}$ Furthermore, in another study, $60 \%$ of lateral incisors and $43 \%$ of central incisors were seen to have pulpal involvement. ${ }^{4}$

\section{Treatment options}

Treatment of resorption cases can be complicated and often requires multidisciplinary input. Treatment is dependant on the level of resorption and prognosis of the resorbed tooth. In cases of resorption the question should be asked as to whether the tooth is so severely damaged that there is danger of losing the tooth during orthodontic treatment. The resorbed tooth can be left, retained as long as possible in preparation for implants or the tooth could be extracted straight away. ${ }^{13}$

Attempts should be made to distance the causative canine from the resorbing teeth as early as possible either by surgical removal or traction. This should be carried out urgently, even before other stages in the treatment plan such as aligning which would normally take precedence, as this will arrest any resorption..$^{36}$ This highlights the importance of identifying resorption as early as possible.

Where the resorbed tooth is vital, even in cases of severe resorption, the resorbed tooth can be maintained without the need 
for root canal treatment. ${ }^{30,34,37-39}$ In such cases where the resorbed incisor is retained, as opposed to extracted, orthodontic alignment only caused a small amount of further resorption. The long term prognosis for these teeth has been shown to be good with no evidence of discolouration, increased mobility or symptoms. ${ }^{36}$

Extraction and substitution of the canine with a prosthesis seems to be the most common means of treating such cases ${ }^{34}$ although retention of the resorbed incisor using dento-alveolar anchor pins has also been described though now very rarely used. ${ }^{28}$

\section{RESTORATIVE DENTISTRY CONSIDERATIONS}

In this article, two cases of severe central incisor resorption are presented where the impacted canine was successfully camouflaged as the central incisor requiring a multidisciplinary approach with restorative dentistry colleagues. For all treatment plans that require input from more than one colleague, the ideal arrangements are that before treatment commences:

- Patients (and parents) meet all members of the clinical team

- Each stage of the treatment plan and the expected outcome of treatment are agreed by all members of the clinical team

- There is agreement about responsibility for each stage: 'who', 'what', 'when', 'where'.

In this clinical situation, the orthodontic treatment stages allow the ectopic canine tooth to be retained and repositioned as a central incisor tooth. As the shape and often the shade of a canine differ from those of a central incisor, it is very likely that restorative dentistry will be required to disguise the tooth. This may be provided either by the patient's general dental practitioner or, by agreement, with another clinician such as a specialist in restorative dentistry.

To disguise the canine as a central incisor, the amount of restorative treatment required will depend on three factors:

1. The degree of difference in shape and shade between the canine tooth and the contra-lateral central incisor tooth

2. The visibility of the anterior teeth during normal function and full smiling
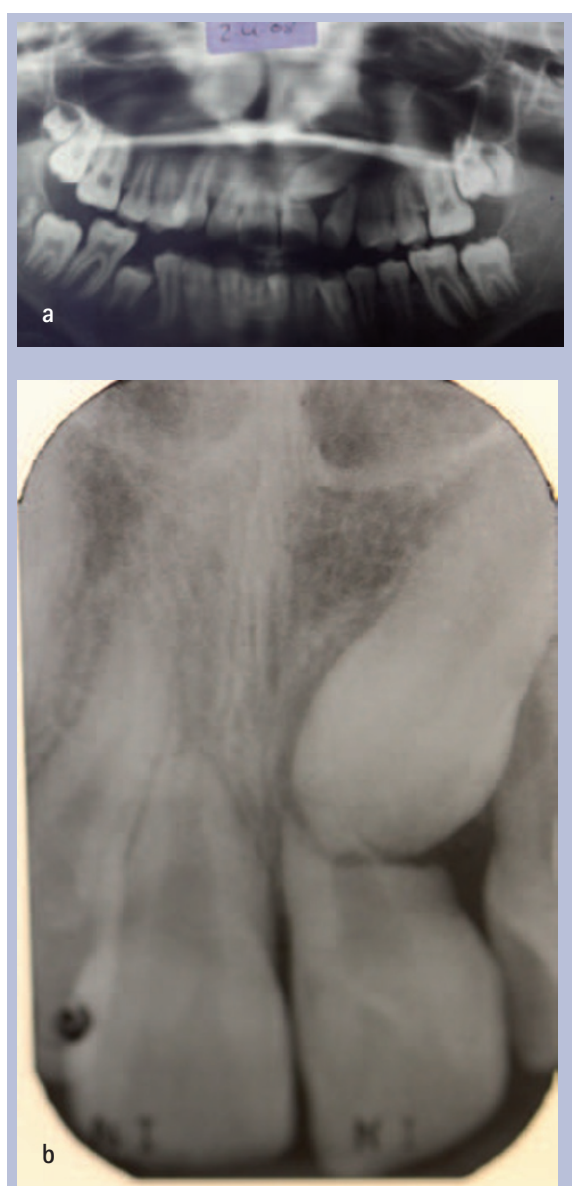

Figs 5a and b An OPG and peri-apical (PA) radiograph is used to identify the position of the canine and the extent of root resorption. The PA reveals extensive resorption of the UL1

3. The patient's personal requests for a final dental appearance that is as close as possible to a natural appearance.

If sufficient care is taken with the final stages of orthodontic canine repositioning, it is possible to disguise the canine tooth and achieve an acceptable aesthetic and functional outcome.

The authors recommend the following considerations.

Gingival margin: the gingival margin should be level with that of the contra-lateral central incisor. The highest point of the gingival margin (the zenith) should ideally be slightly distal to the vertical midline of the tooth. It is possible, using periodontal surgery, to alter the position and shape of the canine gingival margin to match that of the contra-lateral central incisor. However, it is not recommended if this would cause exposure of any of the canine root surface.

Mesio-distal position: the canine will eventually occupy a space of equal width

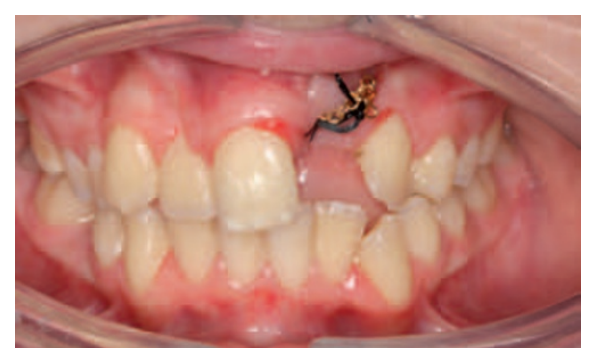

Fig. 6 The resorbed UL1 has been extracted and a gold chain attached to the unerupted canine

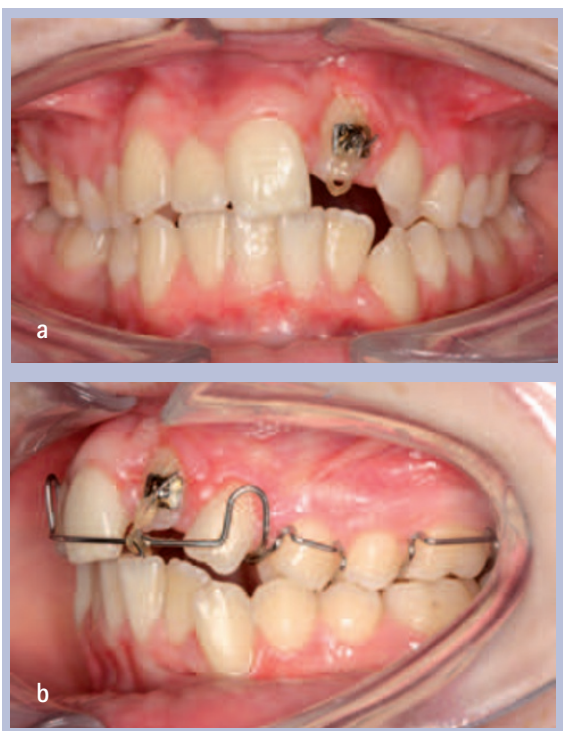

Figs $7 a$ and $b$ Once the UL3 had started to erupt an orthodontic bracket and attachment were placed to begin alignment. An upper removable appliance was used to engage the aligning traction

to the contra-lateral central incisor. If the canine is narrower than the space, it is usually placed in the middle of the space. Occasionally, the shape of the canine gingival margin will dictate that the tooth is placed either slightly more mesially or distally in order to place the canine zenith correctly.

Cervical margin: the cervical margin emerges from the gingival margin and this 'emergence profile' should match that of the contra-lateral central incisor. If a veneer is subsequently to be used to restore the canine, the veneer margin should blend seamlessly with the canine surface, to avoid a positive margin that is both unattractive and unhealthy. The labial position of the canine's cervical margin should therefore match the contra-lateral central incisor before the veneer is placed.

A canine frequently has a greater labiopalatal dimension than a central incisor. Even when the canine is correctly placed to create an appropriate emergence profile, the palatal shape of the canine may 


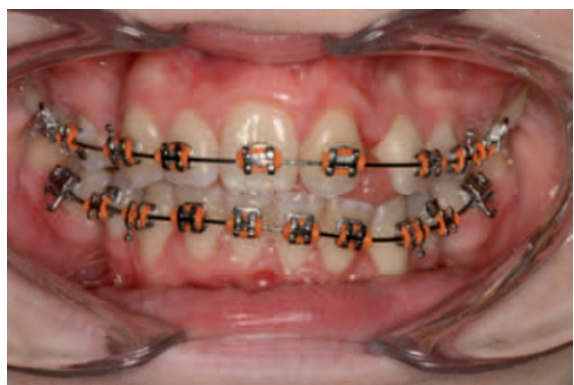

Fig. 8 A full orthodontic fixed appliance was placed to achieve further alignment using initially flexible $\mathrm{Ni}-\mathrm{Ti}$ arch wires

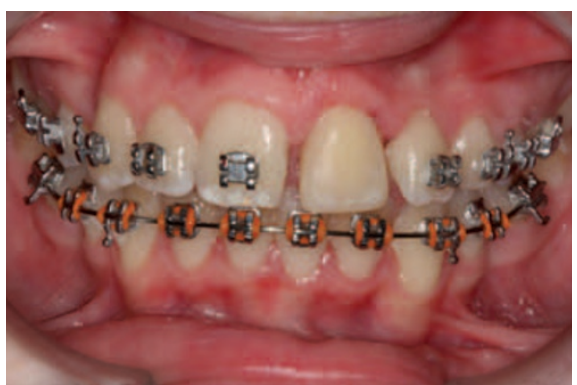

Fig. 9 Once the tooth was in the best possible position the bracket was removed and build-up was undertaken to allow the best possible aesthetic appearance

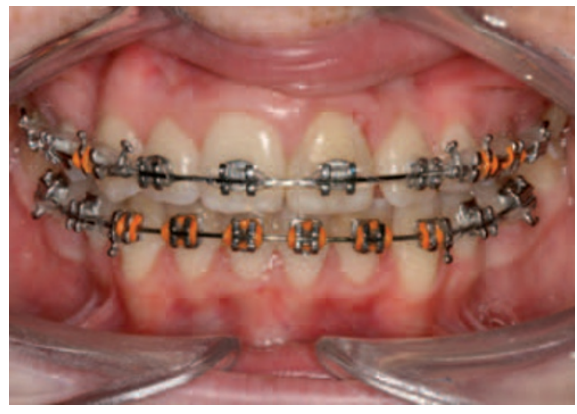

Fig. 10 Once the build-up was completed the orthodontic appliance could be replaced to allow final detailing and positioning of the teeth

cause difficulties in creating an overbite and an appropriate incisal relationship with the opposing lower incisors. The palatal surface of the canine may require subtle reduction.

The incisal relationship is also an important consideration when planning to restore the canine with either a veneer or a composite material. The lower incisors are very likely to remain in contact with the canine tooth and restoration during protrusive movements of the mandible. The final position of the lower incisors, after orthodontic treatment, should therefore allow sufficient room for the restoration in all mandibular movements.

A central incisor generally has two labial planes compared to the more convex,
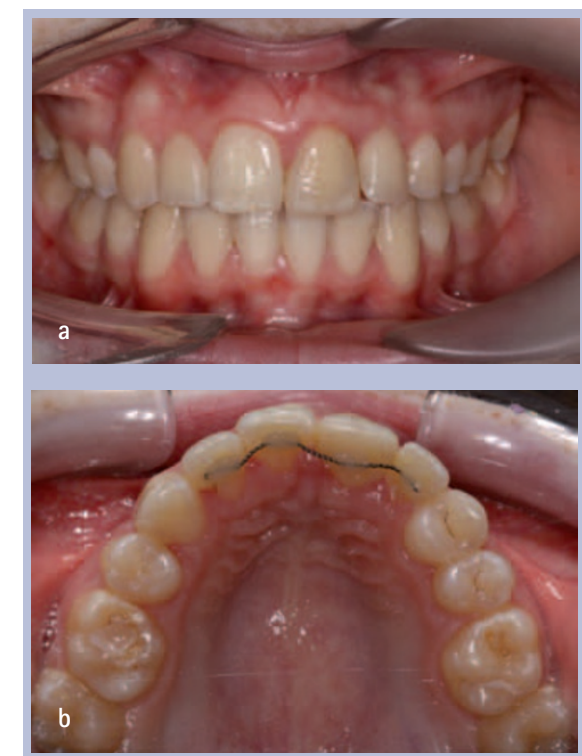

Fig. 11 (a) A nice outcome was achieved with the UL3 camouflaged as the UL1. (b) A bonded retainer was fitted to maintain the corrected positions

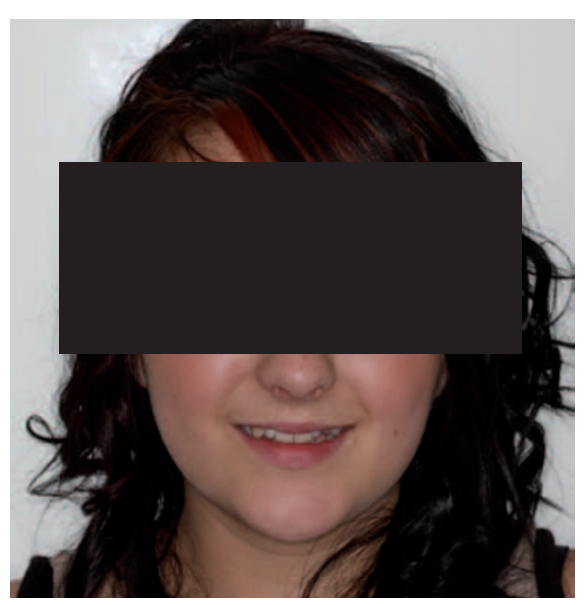

Fig. 12 A 17-year-old female with a Class I skeletal and incisal relationship and a retained upper left deciduous canine (ULC) and ectopic permanent canine

three-plane labial surface of the bulkier canine. The canine is also less translucent and usually a darker shade than the contra-lateral central incisor.

Following orthodontic treatment, the shape and shade of the canine are very likely to need altering and there are various ways, often used in combination, to achieve an aesthetic and functional outcome:

- Single tooth whitening: either in surgery or by tray-based methods

- Removal of the canine tip and flattening of the prominent labial surface

- Addition of composite material to widen the cervical area and to create mesio-incisal and disto-incisal corners to match the contra-lateral central incisor

- Preparation of the tooth and placement of a veneer.

Following restoration, the initial removable retainer will no longer fit. The restorative dentist should liaise very closely with the orthodontist and dental technician so that a new retainer can be made as soon as possible to prevent relapse of the tooth position. Although a fixed palatal retainer, placed at the end of the orthodontic treatment, would overcome this problem, this retainer makes subsequent restoration of the tooth much more challenging and usually compromises the final result.

\section{Case 1}

A 12-year-old patient was referred to the orthodontic department from a local specialist orthodontic practice regarding an ectopic upper left canine (UL3). The orthodontist had diagnosed some resorption to the upper anterior teeth.

On examination the patient presented with a Class II division 1 incisor relationship with a $5 \mathrm{~mm}$ overjet (Figs 4a and b). Intra-orally there was mild to moderate upper and lower arch crowding and the upper left canine was unerupted. Following radiographic examination (Figs 5a and b) the UL3 was overlying both the upper left lateral (UL2) and central (UL1) incisors. There appeared to be extensive resorption to the UL1.

Treatment consisted of extraction of the UL1 along with extraction of the upper right 1st pre-molar, lower left and right 2nd premolars. A gold chain attachment was placed on the unerupted canine (Fig. 6). An upper removable appliance was used to initiate retraction of the canine into the central incisor position (Figs 7a and b). Once this was visible and alignment had a good prognosis a full upper and lower pre-adjusted fixed appliance was placed. The UL3 was further aligned with initial light force arch wires (NiTi), progressing to stainless steel once levelled and aligned (Fig. 8). Once the tooth was in the best position possible the bracket was removed and the restoration placed to further camouflage the UL3 to resemble the extracted UL1 (Fig. 9). This method of placing the restoration, while still in fixed appliances, offers advantages over the conventional approach of completing orthodontic treatment and then undertaking the restoration. 
The restorative dentist has conventionally had to use only the space provided by the orthodontist which can be frequently too large or small. With this method the appliances can be replaced (Fig. 10) once the restoration has been completed and any residual space or further alignment can be undertaken (Fig. 11). It will also allow aesthetic improvements to occur earlier in the treatment improving the patient's experience of treatment. Once any residual space had been closed and the UL3 fully aligned the fixed appliances were removed and a Manchester retainer fitted. ${ }^{40}$ The patient was extremely happy with the outcome.

\section{Case 2}

A 17-year-old female (Fig. 12) was referred by her GDP to assess and provide treatment to an un-erupted upper left canine. Following clinical (Figs 13a and b) and radiographic examination (Fig. 14) the canine was found to be overlying both the lateral (UL2) and central incisors (UL1) with a significant amount of root resorption suspected. The patient was subsequently referred for a cone beam CT scan (Fig. 15) of the area. This revealed extensive resorption to the UL1 and resorption to the UL2 involving 50\% of the dentine but sparing the pulp.

Two treatment plans were discussed involving the placement of the ectopic canine (UL3) in the correct canine position with the ultimate need for replacement of the UL1 and UL2. Alternatively the UL3 could be aligned in the UL1 position accepting ultimate replacement of the UL2 and UL3. The first option was preferred and arrangement to expose and place a gold chain to the UL3 was undertaken. During the alignment phase it was clear that the UL3 was developing towards the UL1 region and a change in treatment plan was agreed, favouring the second option.

An upper fixed appliance was placed and progressed to stainless steel archwires. The UL3 was engaged in the fixed appliance to align using a 'piggy-back' technique (Figs 16a and b). At this stage the UL1 was extracted and remaining root removed (Figs 17a-d). As the UL3 continued to align the crown of the UL1 was gradually reduced to provide vertical alignment space (Fig. 18). Treatment was completed to the UL3 (Fig. 19) and arrangement made to restore the crown of the UL3 to resemble and disguise as the UL1 (Figs 20a-c). The
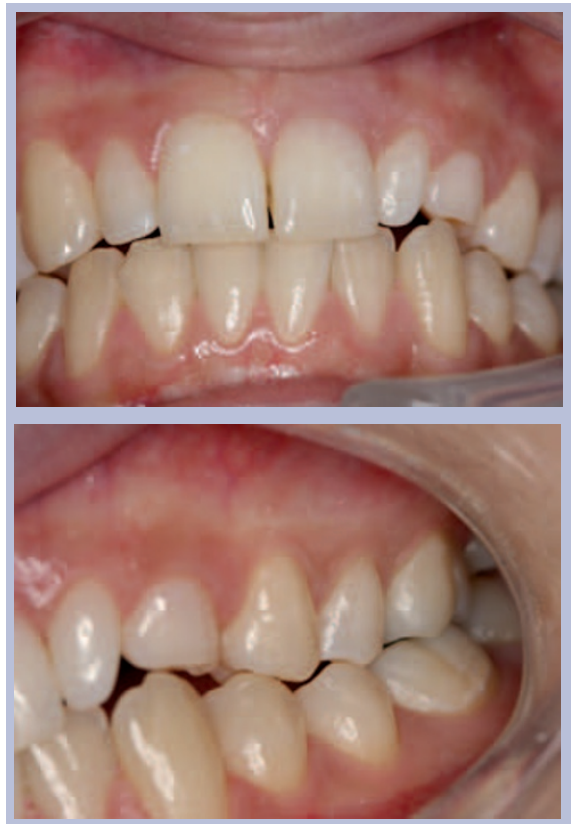

Figs 13a and $b$ The dental views show a diminutive upper left lateral incisor and retained ULC. The molar relationship is Class I bilaterally

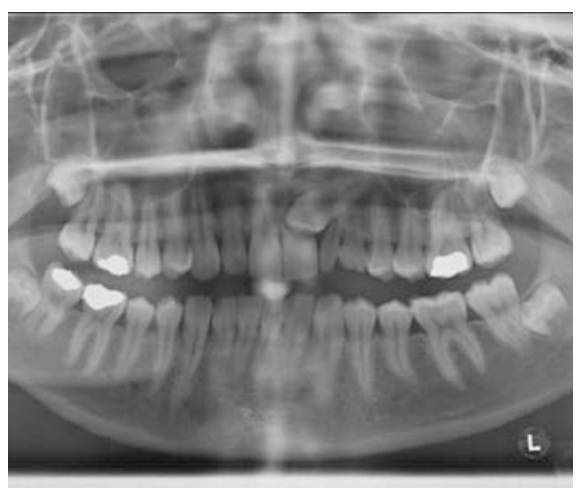

Fig. 14 The OPG radiograph reveals the UL3 to be overlying the UL1 with extensive resorption to this tooth. This is a suspicion of resorption also affecting the UL2. The ULC is retained but with good root morphology

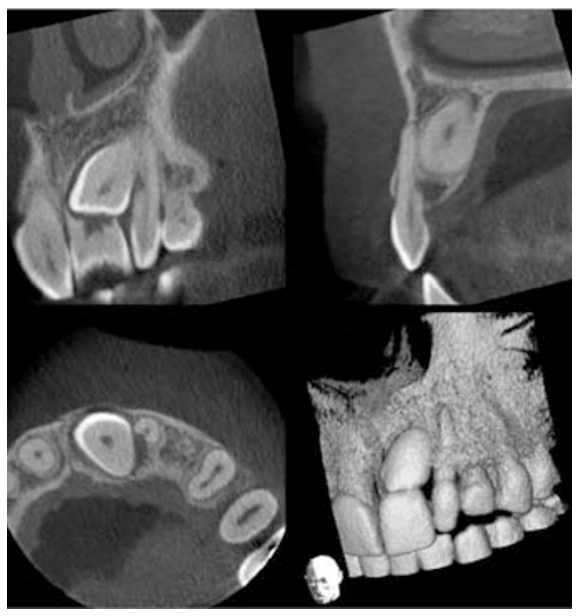

Fig. 15 A cone beam CT scan was taken which indicated complete resorption of the UL1 and also a resorption cavity affecting the mesio-palatal aspect of the UL2 root
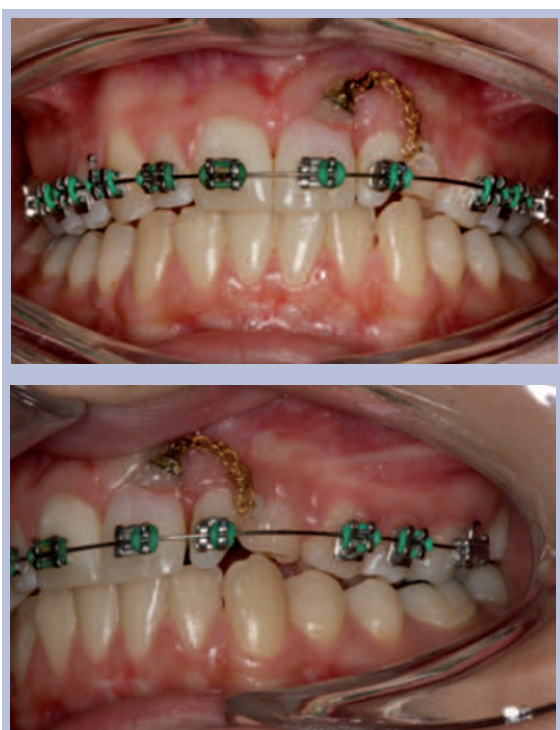

Figs 16a and $b$ The UL3 was exposed and a bracket placed. Resorption of the UL1 had already started to take place
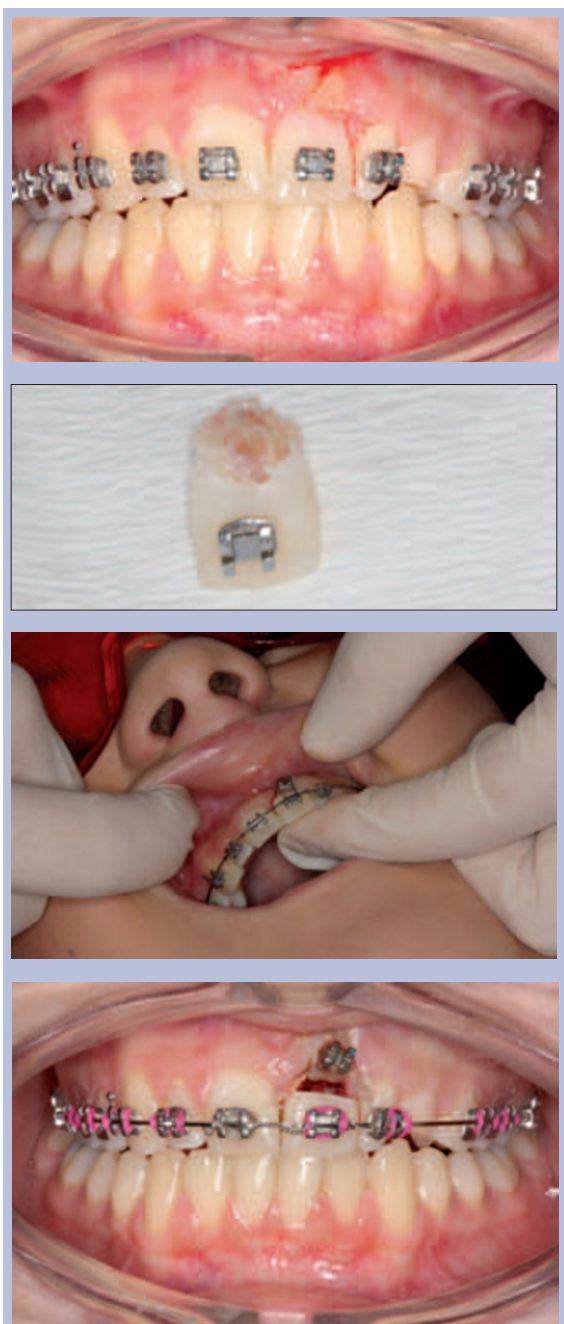

Fig. 17 (a) The UL1 was extracted and a bracket placed on the partially erupted UL3 to start alignment. ( $b$ and $c$ ) To maintain some form of aesthetics the UL1 was modified and replaced back on the archwire (d) Ligature wire ties were engaged to increase stability 


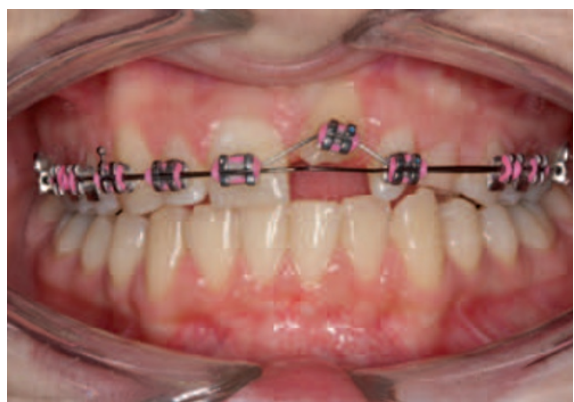

Fig. 18 The UL3 has been fully engaged with a Ni-Ti 'piggy-back'. A stainless steel base arch-wire is used to support the anchorage

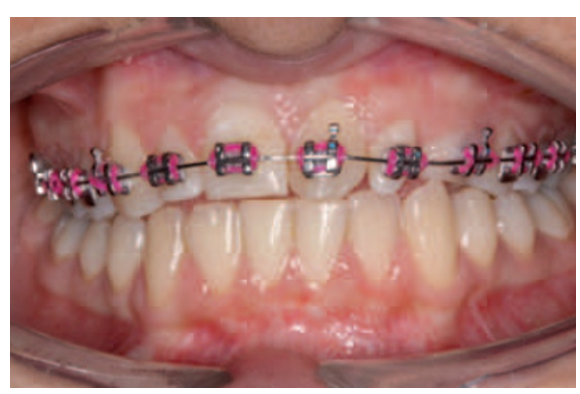

Fig. 19 The UL3 is in a good position and ready for the restorative modifications

upper left retained $\mathrm{C}$ will be left in situ until a replacement is needed. The patient was very happy with the aesthetic outcome in addition to elimination of the need for a prosthetic replacement of her front tooth.

\section{CONCLUSION}

Impacted maxillary canines can cause clinicians to face many difficult treatment challenges. They pose a risk to adjacent teeth such as resorption, particularly the lateral incisor and, less commonly, the central incisor. Early detection and intervention is of paramount importance and therefore close attention must be paid to the eruption of the maxillary canine by all clinicians but particularly the GDP who is likely to see the patient on a regular basis. When the impacted tooth is detected, the position and proximity to adjacent teeth can be further assessed using a variety of radiological methods such as cone beam computed tomography which is particularly helpful where resorption is suspected. In cases where resorption has affected the adjacent teeth, particularly the central incisor, one treatment option is to extract the resorbed tooth and expose the impacted canine to camouflage as the central incisor. To successfully complete this treatment plan, a multidisciplinary approach between the orthodontist and a restorative dentistry colleague is often needed.
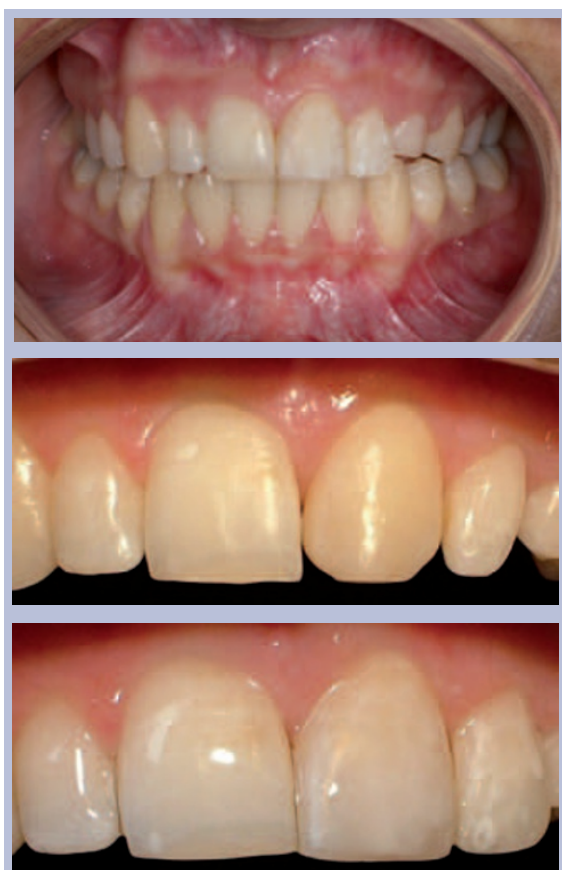

Figs 20a-c The appearance of the UL3 in the UL1 position with restorative modifications

1. Stewart J A, Heo G, Glover K E, Williamson P C, Lam E W, Major P W. Factors that relate to treatment duration for patients with palatally impacted maxillary canines. Am J Orthod Dentofacial Orthop 2001; 119: $216-225$.

2. Ericson S, Kurol J. Radiographic examination of ectopically erupting maxillary canines. Am J Orthod Dentofacial Orthop 1987; 91: 483-492.

3. Walker L, Enciso R, Mah J. Three-dimensional localization of maxillary canines with cone-beam computed tomography. Am J Orthod Dentofacial Orthop 2005; 128: 418-423.

4. Ericson S, Kurol J. Resorption of incisors after ectopic eruption of maxillary canines: a CT study. Angle Orthod 2000; 70: 415-423.

5. Jacoby $\mathrm{H}$. The aetiology of maxillary canine impactions. Am J Orthod 1983; 84: 125-132.

6. Thilander B, Jakobsson S O. Local factors in impaction of maxillary canines. Acta Odontol Scand 1968; 26: 145-168.

7. Brin I, Solomon Y, Zilberman Y. Trauma as a possible etiologic factor in maxillary canine impaction. Am J Orthod Dentofacial Orthop 1993; 104: 132-137.

8. Becker A, Zilberman Y, Tsur B. Root length of latera incisors adjacent to palatally displaced maxillary cuspids. Angle Orthod 1984; 54: 219-225.

9. Lewis B, Durning P, McLaughlin W, Nicholson P T. Canine transposition following trauma and loss of a central incisor: treatment options. J Orthod 2005; 32: $11-19$.

10. Becker A. Palatal canine displacement: guidance theory or an anomaly of genetic origin? Angle Orthod 1995; 65: 95-98.

11. Berkovitz B, Holland G, Moxham B. Oral anatomy, histology and embryology. 3rd ed. p 358. London: Mosby Year Book, 1992.

12. Hurme $\vee 0$. Ranges of normalcy in the eruption of permanent teeth. J Dent Child 1949; 16: 11-15.

13. Bjerklin K, Ericson S. How a computerized tomography examination changed the treatment plans of 80 children with retained and ectopically positioned maxillary canines. Angle Orthod 2006; 76: 43-51.

14. Ericson S, Kurol J. Early treatment of palatally erupting maxillary canines by extraction of the primary canines. Eur J Orthod 1988; 10: 283-295.

15. Power S M, Short M B. An investigation into the response of palatally displaced canines to the removal of deciduous canines and an assessment of factors contributing to favourable eruption. $\mathrm{Br} J$ Orthod 1993; 20: 215-223.
16. Becker A, Shteyer A, Lustman J. Surgical exposure of impacted teeth. In Becker A (ed) The orthodontic treatment of impacted teeth. 2nd ed. p 30. London: Martin Dunitz Ltd, 1998.

17. Fournier A, Turcotle J, Bernard C. Orthodontic considerations in the treatment of maxillary impacted canines. Am J Orthod 1982; 81: 236-239.

18. Kjaer I. Morphological characteristics of dentitions developing excessive root resorption during orthodontic treatment. Eur J Orthod 1995; 17: 25-34.

19. Becktor K B, Steiniche K, Kjaer I. Association between ectopic eruption of maxillary canines and first molars. Eur J Orthod 2005; 27: 186-189.

20. Ericson S, Kurol J. Incisor resorption caused by max illary cuspids - a radiographic study. Angle Orthod 1987; 57: 332-346.

21. Heimisdottir K, Bosshardt D, Rufc S. Can the severity of root resorption be accurately judged by means of radiographs? A case report with histology. Am J Orthod Dentofacial Orthop 2005; 128: 106-109.

22. Ericson S, Kurol J. Incisor root resorption due to ectopic maxillary canines imaged by computerized tomography. A comparative study in extracted teeth. Angle Orthod 2000; 70: 92-99.

23. Elefteriadis J, Athanasiou A. Evaluation of impacted canines by means of computerized tomography. Int Adult Orthodon Orthognath Surg 1996; 11: 257-264.

24. Pauwels R, Beinsberger J, Collaert B et al. Effective dose range for dental cone beam computed tomography scanners. Eur J Radio/ 2012; 81: 267-271.

25. European Commission. Radiation protection 136. European guidelines on radiation protection in dental radiology. Luxembourg: Office for Official Publications of the European Communities, 2004

26. Ludlow J B, Davies-Ludlow L E, Brooks S L, Howerton W B. Dosimetry of 3 CBCT devices for oral and maxillofacial radiology: CB Mercuray, NewTom $3 \mathrm{G}$ and i-CAT. Dentomaxillofac Radiol 2006; 35: 219-226.

27. Tronstad L. Root resorption - aetiology, terminology and clinical manifestations. Endod Dent Traumatol 1988; 4: 241-252.

28. Sasakura H, Yoshida T, Murayama S, Hanada K, Nakajima T. Root resorption of upper permanent incisor caused by impacted canine. An analysis of 23 cases. Int J Oral Surg 1984; 13: 299-306.

29. Knight $\mathrm{H}$. Tooth resorption associated with the eruption of maxillary canines. Br J Orthod 1987; 14: 21-31.

30. Rimes R J, Mitchell C N T, Willmot D R. Maxillary incisor resorption in relation to the ectopic canine: a review of 26 patients. Eur J Orthod 1997; 19: 79-84.

31. Ericson S, Kurol J. Resorption of maxillary lateral incisors caused by ectopic eruption of the canines. Am J Orthod Dentofacial Orthop 1988; 94: 503-513.

32. Ericson S, Kurol J, Falahat B. Does the canine dental follicle cause resorption of permanent incisor roots? A computed tomographic study of erupting maxillary canines. Angle Orthod 2002; 72: 95-104.

33. Brin I, Becker A, Zilberman Y. Resorbed lateral incisors adjacent to impacted canines have normal crown size Am J Orthod Dentofacial Orthop 1998; 104: 60-66.

34. Saldarriaga J R, Patiño M C. Ectopic eruption and severe root resorption. Am J Orthod Dentofacial Orthop 2003; 123: 259-265.

35. Arens D E. An alternative treatment for severely resorbed maxillary lateral incisor: a sequela of ectopic eruption. J Endod 1995; 21: 95-100.

36. Becker A, Chaushu S. Long-term follow-up of severely resorbed maxillary incisors after resolution of an etiologically associated impacted canine Am J Orthod Dentofacial Orthop 2005; 127: 650-654.

37. Savage R R, Kokich V G. Restoration and retention of maxillary anteriors with severe root resorption. J Am Dent Assoc 2002; 133: 67-71.

38. Parker W S. Root resorption - long-term outcome. Am J Orthod Dentofacial Orthop 1997; 112: 119-123.

39. Shellhart W C, Jasper S, Abrams H, Wilson T. Case report: management of significant root resorption associated with maxillary canine impaction. Angle Orthod 1998; 2: 187-192.

40. Waring D T, Cappelli R. The Manchester positioner - dual retention made easy. J Orthod 2009; 36: 111-114. 